\title{
Effect of Write Current Waveform on Magnetization and Head-Field Dynamics of Perpendicular Recording Heads
}

\author{
Werner Scholz and Sharat Batra \\ Seagate Research, Pittsburgh, PA 15222-4215 USA
}

\begin{abstract}
The response of perpendicular magnetic recording heads with a single turn and two turn coils is calculated using a full micromagnetic model, including return pole and soft underlayer. We study the effect of coil current waveforms with different rise times and overshoots. For fast coil current rise times, the head field shows very little response, until the coil current changes its polarity and it is limited by the intrinsic magnetization dynamics. Even the effect of overshoot is limited by the same mechanism. Shorter yoke length and coil turns close to the air-bearing surface improve the head-field dynamics.
\end{abstract}

Index Terms-Coil current, head-field dynamics, perpendicular magnetic recording, write head.

\section{INTRODUCTION}

$\mathbf{T}$ HE continuing advances in magnetic recording density and data rates require an ever more detailed understanding and control of the dynamic processes in magnetic write heads, recording media, and read heads. As the typical dimensions of the write pole of single-pole-type perpendicular recording heads shrink to about $100 \mathrm{~nm}$ and data rates exceed $1 \mathrm{~Gb} / \mathrm{s}$, the dynamic magnetization processes in the write pole and its interaction with the soft underlayer (SUL) and the return pole become very important [1]-[3]. In turn, the reduced size of the recording head makes it possible to perform fully micromagnetic simulations [4]-[6], which can model the intrinsic magnetization dynamics and the dynamic interaction with the SUL, which has to be considered part of the head because of its mirroring effect. However, the SUL should not be treated as a simple mirror image, but the magnetization dynamics in the SUL should also be modeled using the Landau-Lifshitz-Gilbert equation of motion [1], [4]. Thus, in order to calculate the head field (i.e., the response of the recording head to the exciting coil currents) accurately, full micromagnetic simulations are required.

\section{MicRomagnetic Model}

We have used a large-scale finite-element micromagnetics model [6] to study the head-field dynamics of single-pole-type perpendicular recording heads [7] (see Fig. 1).

The head geometry and material parameters have been described in detail in [6]. The model includes the write pole, yoke, return pole, and SUL, which are discretized with tetrahedral finite elements with first-order linear basis functions. The current density distribution in the coils and the resulting magnetic field are calculated with Flux3D [8], and then the magnetic field is imported in the micromagnetic model as a simple field map. Due to the linearity of the Biot-Savart law, the field map can be scaled according to the current waveform. However, any eddy

Digital Object Identifier 10.1109/TMAG.2006.878632

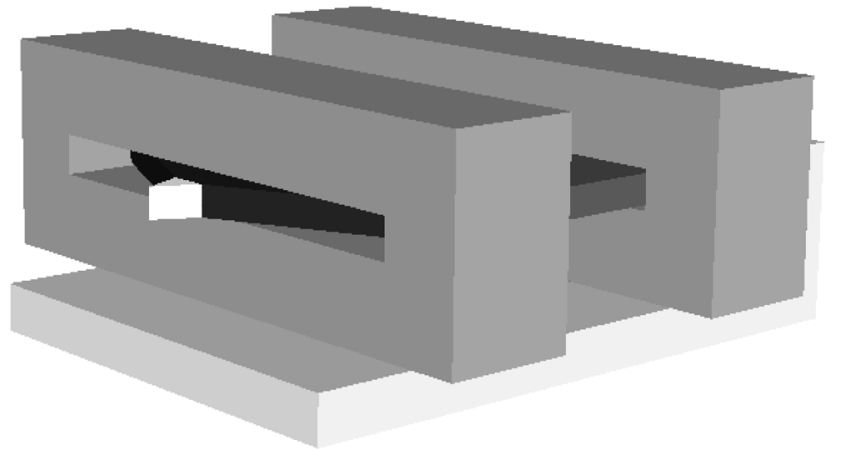

Fig. 1. 3-D model of a perpendicular recording head with a two-turn coil. The two-turn helical coil (simplified to two closed turns) wraps around the write pole. The return pole is shown on the bottom. The SUL is fully discretized and treated micromagnetically but not shown in this figure.

current effects in the coils or in the head, as well as any thermal effects, have been neglected.

The magnetization dynamics are calculated according to the Landau-Lifshitz-Gilbert equation of motion

$\frac{d \mathbf{M}}{d t}=-\frac{|\gamma|}{1+\alpha^{2}}(\mathbf{M} \times \mathbf{H})-\frac{\alpha|\gamma|}{|\mathbf{M}|\left(1+\alpha^{2}\right)}(\mathbf{M} \times(\mathbf{M} \times \mathbf{H}))$

and the effective magnetic field $\mathbf{H}$ is calculated from the contributions of magnetostatic fields, magnetocrystalline anisotropy, exchange interactions, and external magnetic fields. The damping constant $\alpha$ is assumed to be 0.2 in all magnetic parts of the model [6], and together with the exclusion of eddy currents, this provides a best-case scenario.

\section{RESULTS}

In order to study the magnetization and, as a result of that, the field dynamics of perpendicular recording heads with micromagnetic simulations, the head and SUL have to be initialized with a realistic magnetization distribution. We start with a homogeneous magnetization distribution parallel to the crosstrack direction, and apply an energy-minimization method to quickly relax the magnetization to a possible remanent state. All dynamic simulations start from this remanent state, 


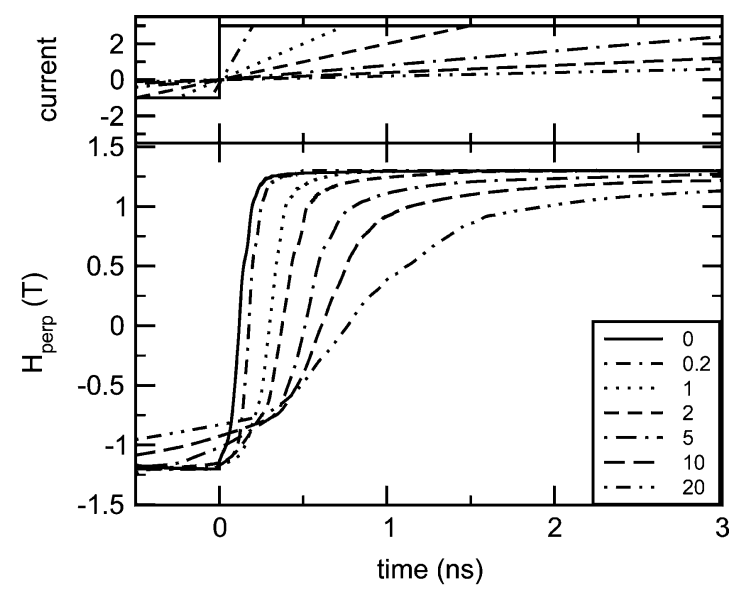

Fig. 2. Time dependence of the perpendicular field component of a single-pole-type head for different current rise times (in units of ns in the legend). The write current in the top graph is given in units of $150 \mathrm{~mA}$, and switches from $-150 \mathrm{~mA}$ to $+450 \mathrm{~mA}$, with the rise times given in the legend.

although the magnetization distribution of this remanent state is still somewhat arbitrary. In order to make sure that the dynamic behavior is credible and reproducible, the write heads are "preconditioned" with a $1 \mathrm{~Gb} / \mathrm{s}$ waveform for a few transitions before the actual data are collected. Then, we studied the effect of current rise time $t_{r}$, current overshoot, overshoot duration $t_{\text {dur }}$, and head geometry (yoke length, coil position) on the magnetization dynamics in the write pole and the head field.

Fig. 2 shows the field dynamics for different current rise times $t_{r}$. After a few preconditioning cycles, the recording head is equilibrated with a steady-state coil current of $-150 \mathrm{~mA}$ through the single-turn coil. Then the current is ramped linearly from this value to $+450 \mathrm{~mA}$ (corresponding to a $200 \%$ overshoot) with different rise times, and the coil current is kept steady at this high value to drive the head hard into saturation and avoid effects of the overshoot duration.

In Fig. 2, the current waveforms and head-field traces have been shifted, such that the coil current is zero at $t=0$. This reveals that the head field shows quite little response until the coil current reverses its polarity and actively tries to switch the magnetization. As a result, the head field lags behind the coil current, and we can distinguish two different regimes. For slow rise times ( $>2 \mathrm{~ns}$ ), there is some decay in the head field (for $t<0$ in Fig. 2), because the head has plenty of time to relax its magnetization toward the remanent state at low coil currents. For fast current rise times, the magnetization dynamics is slower than the change in coil current, and the head field remains at the value of its steady state until the coil current changes its sign.

For very fast current rise times $(\leqslant 0.2 \mathrm{~ns})$, the head-field response is almost independent of the current rise time, because the magnetization dynamics in the head is too slow to respond to the fast change in coil current. Thus, the head-field dynamics is determined by the intrinsic response time of the magnetization, which has been shown to depend on the Gilbert damping parameter [4], [6], [9]. For very slow rise times (>1 ns), we find a strong influence on the head-field response and its rate of change, which has been shown to influence the transition location and curvature [10].

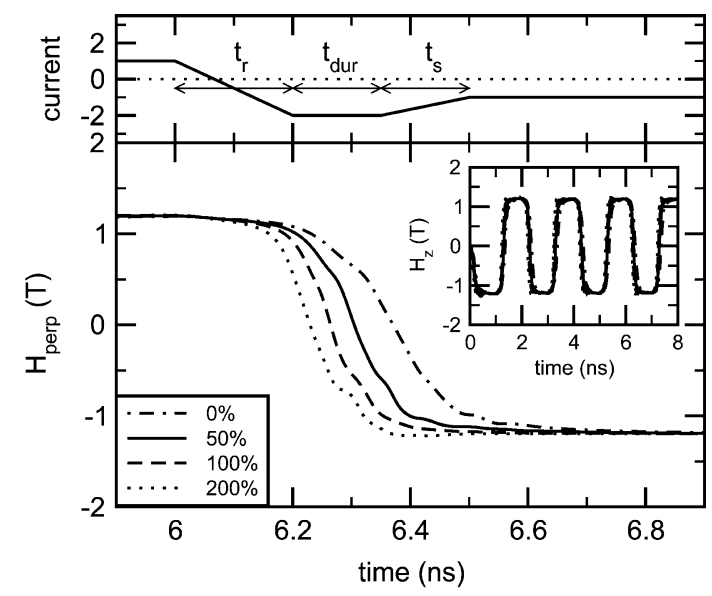

Fig. 3. Time dependence of the perpendicular field component of a single-pole-type head for different overshoot levels $(0 \%, 50 \%, 100 \%, 200 \%$, with 0.2 ns current rise time). In all cases, the coil current starts changing at $t=6 \mathrm{~ns}$. The inset shows the head-field dynamics of the complete simulation. Only one write current waveform ( $t_{r}=0.2 \mathrm{~ns}, 100 \%$ overshoot) is shown in the top graph. The settling time $t_{s}$ has been set to $0.15 \mathrm{~ns}$ in all simulations.

Typically, the current rise time in magnetic recording systems is on the order of $0.2 \mathrm{~ns}$, which puts the head into the regime of fast rise times. Fig. 3 shows the effect of current overshoot for such a system. In this set of simulations, we observe a shift of the head-field traces, which is due to the different times when the coil current changes its polarity. In this plot, all coil currents start to change at $t=6 \mathrm{~ns}$, but due to the different overshoot levels, they switch from positive to negative current at different times. Thus, the head-field traces are shifted. In addition, the head-field rise time depends on the overshoot level, which determines how hard the head is driven. There is a significant difference in the head-field response $d H_{\text {perp }} / d t$ at low overshoot levels ( $\leqslant 50 \%$ ), where the added driving force of the coils (their Oersted field) speeds up the switching of the magnetization. This effect diminishes for large overshoots $(\geqslant 100 \%$, where the switching time of the magnetization does not decrease significantly with an increasing magnetic field. In the absence of any overshoot, the head field changes its sign 0.37 ns after the coil current starts its rise time (at $t=6 \mathrm{~ns}$ in Fig. 3). For $50 \%$ overshoot, the head field changes its sign 0.304 ns after the coil current. For a $100 \%$ overshoot, this lag of the head-field response is reduced to $0.264 \mathrm{~ns}$, and for $200 \%$ overshoot, to $0.229 \mathrm{~ns}$.

Fig. 4 shows the effect of current rise time and overshoot for fast current pulses. If the current rise time is faster than the headfield rise time, the head-field response is the same, and only shifted due to a different time when the coil current switches its polarity. In fact, the three different curves for $0 \%$ overshoot and $t_{r}=\{0,0.1,0.2 \mathrm{~ns}\}$ fall on top of each other, if they are shifted to the left by $\{0,0.5,0.1 \mathrm{~ns}\}$, respectively. Yet, the case with $100 \%$ overshoot is not only faster because its coil current switches its polarity earlier, but it also shows a steeper slope, corresponding to a faster head-field response.

The faster current rise time is more effective in improving the head response, because the magnetization in the write poles does not respond to the change in coil current until the coil current changes its polarity and generates a field, which can reverse the magnetization of the write head. 


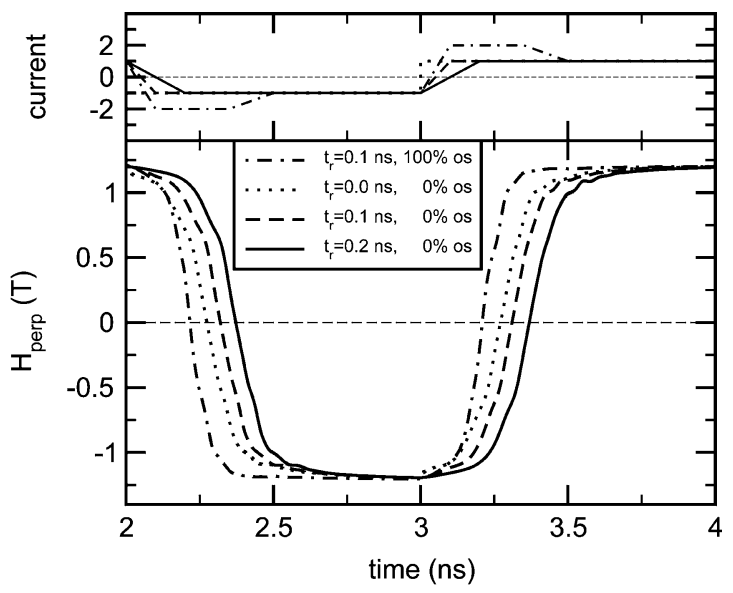

Fig. 4. Time dependence of the perpendicular field component of a single-pole-type head for different current rise times and overshoot. The values in the legend are in units of $\mathrm{A} / \mathrm{ns}$. The write current in the top graph is given in units of $150 \mathrm{~mA}$, and switches from $-150 \mathrm{~mA}$ to $+450 \mathrm{~mA}$ with the rise times given in the legend.

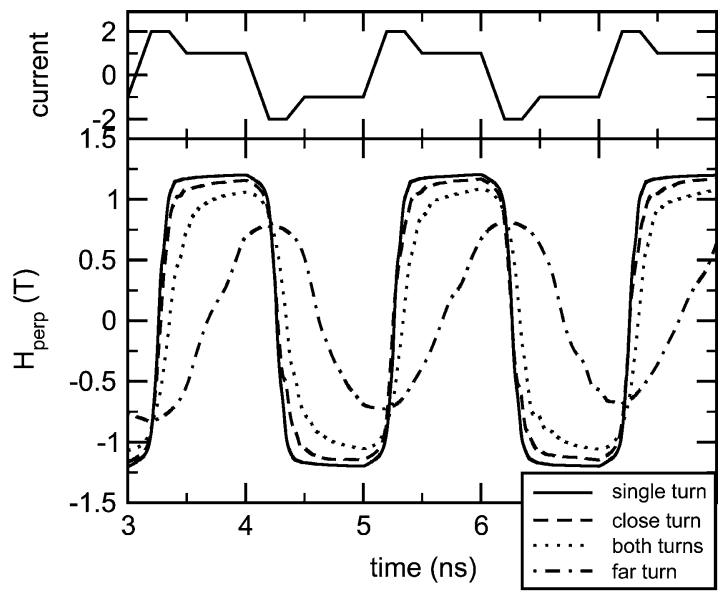

Fig. 5. Head-field dynamics for a single-turn head with short yoke and a two-turn head with longer yoke. In all simulations, the head has been driven with $150 \mathrm{~mA}$ turns, i.e., $150 \mathrm{~mA}$ through the coil of the single-turn head and when only one turn of the two-turn head was used, and $75 \mathrm{~mA}$ through each turn, when both turns of the two-turn head were used.

Finally, Fig. 5 shows a comparison of the head response of a head with short yoke and a single-turn coil and a head with longer yoke and a two-turn coil. The response of the two-turn coil is the slowest, and generates the lowest field when the second turn (far away from the air-bearing surface) carries a current of $150 \mathrm{~mA}$. The flux does not have enough time to propagate to the pole tip and saturate it [11]. The head field changes its sign $0.67 \mathrm{~ns}$ after the coil current starts its rise time, and the maximum head field is reduced by about $33 \%$. If both turns carry a current of $75 \mathrm{~mA}$ (leading to $150 \mathrm{~mA}$ - turns), the head response improves significantly, and the head field changes its sign $0.34 \mathrm{~ns}$ after the coil current. If only the turn close to the air-bearing surface carries a current of $150 \mathrm{~mA}$, we find the fastest response of the head with the two-turn coil. The head field lags only $0.26 \mathrm{~ns}$ behind the coil current and reaches the highest write field of $1.2 \mathrm{~T}$. Yet, in comparison with the head with short yoke and single-turn coil, the approach of the head field to its saturation value is slowed down due to the longer yoke.

\section{CONCLUSION}

We have calculated the head-field response as a function of coil current, overshoot, and head geometry. The head-field delay is reduced by fast current rise times and large overshoots, but it is limited by the intrinsic magnetization dynamics. Short yoke structures and coils with few turns close to the air-bearing surface improve the head response, too.

Thus, in order to design high-performance perpendicular recording heads, which are capable of writing high coercivity media at high data rates, a carefully optimized design is required, which also considers the effects of the write current waveform on the head-field dynamics presented in this paper.

\section{REFERENCES}

[1] M. Schabes, B. Lengsfield, and T. Schrefl, "Micromagnetic modeling of soft underlayer magnetization processes and fields in perpendicular magnetic recording," IEEE Trans. Magn., vol. 38, no. 4, pp. 1670-1675, Apr. 2002.

[2] K. Z. Gao and H. N. Betram, "3-D micromagnetic simulation of write field rise time in perpendicular recording," IEEE Trans. Magn., vol. 38, no. 9, pp. 2063-2065, Sep. 2002.

[3] J. Heidmann, H. Do, M. Xiao, K. Takano, and Y. Ikeda, "Direct measurement of magneto-dynamics in a perpendicular recording system," IEEE Trans. Magn., vol. 42, no. 2, pp. 166-170, Feb. 2006.

[4] K. Takano, "Micromagnetic-FEM models of a perpendicular writer and reader," IEEE Trans. Magn., vol. 41, no. 2, pp. 696-701, Feb. 2005.

[5] M. E. Schabes, T. Schrefl, D. Suess, and O. Ertl, "Dynamic micromagnetic studies of anisotropy effects in perpendicular write heads," IEEE Trans. Magn., vol. 41, no. 10, pp. 3073-3075, <Oct. 2005.

[6] W. Scholz and S. Batra, "Micromagnetic modeling of head field rise time for high data-rate recording," IEEE Trans. Magn., vol. 41, no. 2, pp. 702-706, Feb. 2005.

[7] S. Batra, J. D. Hannay, H. Zhou, and J. S. Goldberg, "Investigations of perpendicular write head design for $1 \mathrm{~Tb} / \mathrm{in}^{2}$," IEEE Trans. Magn., vol. 40, no. 1, pp. 319-325, Jan. 2004.

[8] Magsoft Corp., Flux3D, Troy, NY.

[9] O. Ertl, T. Schrefl, D. Suess, and M. Schabes, "Influence of the Gilbert damping constant on the flux rise time of write head fields," J. Magn. Magn. Mater, vol. 290-291, pp. 518-521, Apr. 2005.

[10] W. Scholz and S. Batra, "Micromagnetic simulation of head-field and write bubble dynamics in perpendicular recording," IEEE Trans. Magn., vol. 41, no. 10, pp. 2839-2844, Oct. 2005.

[11] M. Covington, T. M. Crawford, and G. J. Parker, "Time-resolved measurement of propagating spin waves in ferromagnetic thin films," Phys. Rev. Lett., vol. 89, p. 237202, 2002.

Manuscript received March 13, 2006 (e-mail: werner.scholz@ seagate.com). 